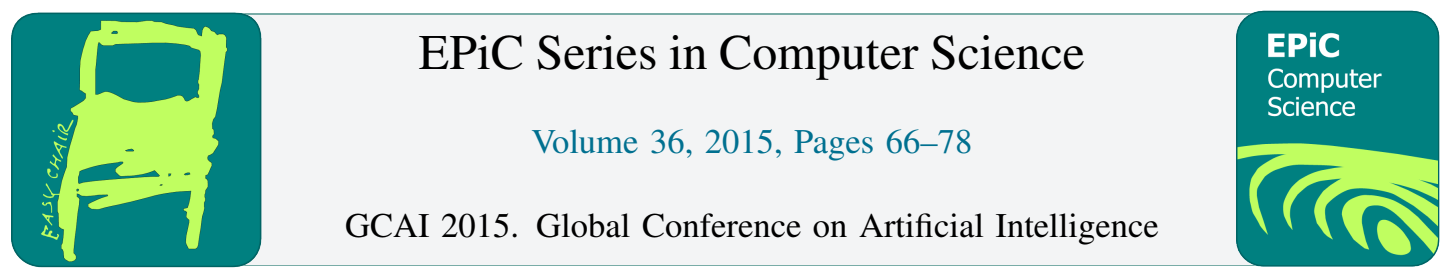

\title{
Linear Models of Computation and Program Learning
}

\author{
Michael Bukatin ${ }^{1}$ and Steve Matthews ${ }^{2}$ \\ 1 Nokia Corporation \\ Burlington, Massachusetts, USA \\ bukatin@cs.brandeis. edu \\ 2 Department of Computer Science \\ University of Warwick \\ Coventry, UK \\ Steve.Matthews@warwick.ac.uk
}

\begin{abstract}
We consider two classes of computations which admit taking linear combinations of execution runs: probabilistic sampling and generalized animation. We argue that the task of program learning should be more tractable for these architectures than for conventional deterministic programs. We look at the recent advances in the "sampling the samplers" paradigm in higher-order probabilistic programming. We also discuss connections between partial inconsistency, non-monotonic inference, and vector semantics.
\end{abstract}

\section{Introduction}

One of the key challenges of program learning is that software tends to be too brittle and insufficiently robust with respect to minor variation.

Biological systems tend to be much more flexible and adaptive with respect to variation. In particular, biological cells are capable of functioning at wide ranges of the level of expression of various proteins, which are machines working in parallel. Regulation of the level of expression of specific proteins is a key element of flexibility of biological systems. It is argued in evolutionary developmental biology that the flexible architecture together with conservation of core mechanisms is crucial for the observed rate of biological evolution [16, 22]. It is suggested that morphology evolves largely by altering the expression of functionally conserved proteins [12].

To incorporate regulation of expression into a system of genetic programming one might evolve programs describing systems of parallel computational processes. Then one might take the CPU allocation and other computational resources given to a particular computational process as computational equivalent of the level of expression of a particular protein.

Of course, many of the architectures for parallel computations are brittle as well, with delicate mechanisms of writing to shared memory and locks. To achieve flexibility one should use parallel architectures which minimize those delicate interdependencies.

Computational architectures which admit the notion of linear combination of execution runs are particularly attractive in this sense. Then one can regulate the system simply by controlling coefficients in a linear combination of its components. 
In this paper we consider two computational architectures which admit linear combinations of execution runs.

One such architecture is probabilistic sampling. If one has two samplers generating points of two distributions with a uniform speed, so that the notion of "a number of points generated per unit of time" is well defined for both of them, one can obtain a sampler generating a linear combination of those two distributions with arbitrary positive coefficients simply by running those two samplers in parallel with appropriate relative speeds.

We argue that it should be easier to learn probabilistic programs than to learn deterministic programs due to the fact that probabilistic programs admit linear combinations of execution runs. We also discuss the techniques to allow negative coefficients in those linear combinations later in the text.

There is a lot of affinity between methods of evolutionary programming and methods of probabilistic sampling. Evolutionary schemas can be considered as particular sampling methods, while many sampling schemas have strong evolutionary flavor (more details in Section 2).

This means that instead of thinking in terms of genetic programming for probabilistic programs one might think about program learning in terms of the "sampling the samplers" paradigm, namely in terms of probabilistic programs sampling other probabilistic programs (generative models producing other generative models as their points). This "sampling the samplers" paradigm manifests itself, in particular, in recent work on learning probabilistic programs by Yura Perov and Frank Wood [33] and also in recent advances in compositional concept learning obtained by Brenden Lake [24]. We review these and some other recent advances in higher-order probablistic programming in Section 3.

\subsection{Negative Coefficients}

Another computational architecture which admits linear combinations of execution runs is generalized animation. We define a generalized (monochrome) image as a map from a set (called the set of points) to reals. We define a generalized (monochrome) animation as a map from time (discrete or continuous) to generalized images. Linear combination of images is defined point-wise. The secondary structure on the set of points might vary. To obtain a conventional monochrome image the secondary structure typically assigns coordinates from a discretized rectangle to points. For display purposes, zero is normally associated with gray level in the middle between the most dark and the most bright possible values. This approach allows the use of both positive and negative coefficients in the linear combinations of generalized images and animations.

Conventional color images and music are examples of generalized animations, and the use of linear combinations of those is standard in video and audio mixing software.

One feature shared between animations and probabilistic programs is that complex behaviors can often be expressed by very short programs. Many software experiments including our own work with simulated reflection in water waves $[9,15]$ demonstrate that interesting and expressive dynamics can result from simple programs.

Another feature animations seem to share with sampling architecture is that they tend to be nonbrittle, and that their mutations and crossover tend to produce meaningful results in the evolutionary setting [13]. This architecture provides a direct way to incorporate aesthetic criteria into software systems. This architecture can also leverage existing animations, digital and physical (such as light refections and refractions in water), as computational oracles.

A lot of expressive power of this architecture comes from the ability to have non-standard secondary structures on the set of points. Points can be associated with vertices or edges of a graph, grammar rules, positions in a matrix, etc. One should be able to formulate mechanisms of higher-order animation programming via variable illumination of elements of such structures. 


\subsection{Negative Probability}

In order to enable both positive and negative coefficients for probabilistic sampling one can allow sampling via two parallel sampling channels: a positive one and a negative one.

There is evidence that signed functions are sampled via parallel positive and negative channels in neural system, for example, in retina (see pages 65 and 173 of [29]). The idea that some of the brain functioning might be understood as Markov Chain Monte Carlo sampling was developed in recent years and led to fruitful applications to the computational schemes robust with respect to noise and benefiting from presence of noise and thus suitable for implementation in low-powered circuits (see [27] and references therein). The combination of this idea and of the evidence for sampling via parallel positive and negative channels is suggestive.

One way to understand and formalize this situation is via allowing negative values for probabilities and probability densities. Quasiprobability distributions allowing both positive and negative probability values have long history. Their first prominent use comes in phase space formulation of quantum mechanics via Wigner quasiprobability distribution in 1940s [31, 18]. The intuition behind the notion of negative probability is discussed in detail in [14]. More recently, negative probabilities are finding use in quantum algorithms [30].

In denotational semantics of probabilistic programs, Dexter Kozen found it fruitful to replace the space of probability distributions with the space of signed measures [23]. This allowed him to express denotations of probabilistic programs as continuous linear operators with finite norms. The probabilistic powerdomain was embedded into the positive cone of the resulting Banach lattice.

\subsection{Partial Inconsistency, Non-monotonic Inference, and Vector Semantics}

Addition of the elements expressing partial degrees of contradiction results in an embedding of an approximation domain into a vector space in yet another important case, the interval numbers, by extending them with pseudosegments $[a, b]$ with the contradictory property that $b<a$.

The resulting spaces tend to be equipped with two Scott topologies dual to each other, which enables both upwards and downwards computable inference steps, and thus facilitates non-monotonic reasoning.

The resulting mathematical landscape is a field directly adjacent to the main topic of this paper. We review this field and present some of our own results there in Section 4.

\subsection{Almost Continuous Transformations of Dataflow Programs}

Because probabilistic sampling and generalized animation are both stream-based, dataflow programming is a natural framework for this situation. Dataflow architecture is convenient for program learning, because syntax of dataflow programs would typically be more closely related to their semantics than the syntax of more conventional programs.

The ability to take linear combinations of execution runs allows us to introduce the notion of almost continuous transformation of dataflow programs [9]. This architecture is applicable to probabilistic sampling and to generalized animation. We implemented an open source software prototype demonstrating the use of those techniques for ordinary animations [15].

This architecture allows us to evolve dataflow programs in almost continuous fashion while those evolving programs are running. This makes it possible to sample almost continuous trajectories in the space of dataflow programs, in addition to the usual practices of sampling the syntax trees of programs, thus enabling new evolutionary and probabilistic schemas of program learning. 


\subsection{Dataflow Graphs as Matrices}

Adopting a discipline of bipartite graphs linking nodes obtained via general transformations and nodes obtained via linear transformations makes it possible to develop and evolve dataflow programs over these classes of computations by continuous program transformations. The use of bipartite graphs allows us to represent the dataflow programs from this class as matrices of real numbers and evolve and modify programs by continuous change of these numbers [10].

The representation of programs as matrices of real numbers makes the task of program learning more similar to the task of machine learning for more narrow and conventional classes of models.

\section{Parallels between Methods of Evolutionary Programming and Probabilistic Sampling}

The connections between probabilistic programming and genetic programming are much tighter than it is usually acknowledged.

Many variants of MCMC are evolutionary in spirit. Acceptance/rejection of the samples corresponds to selection. Production of new samples via modifications of the accepted ones corresponds to mutations to produce offspring from the survivors.

The Bayesian Optimization Algorithm changes the procedure of producing the next generation in genetic algorithms from pairwise crossover to resampling from the estimated distribution of the individuums selected for fitness [32]. This scheme of crossover is used by the seminal MOSES system [26]. This is similar in spirit to population-based methods of sampling.

\section{Some Recent Advances in Higher-Order Probabilistic Program- ming}

We are seeing very rapid progress in probabilistic programming in recent years.

What particularly catches our attention is a series of results solving various computer vision problems as Bayesian inverse problems to computer graphics rendering, starting with [28].

For an example of a powerful model learning scheme for probabilistic programs using matrix decomposition and a context-free grammar of models see [19].

The term "higher-order probabilistic programming" usually means a higher-order functional programming language implementing sampling semantics. Recently we are seeing examples of research implementing higher-order sampling schemas in a more narrow and focused sense of the word: samplers which generate other samplers, probabilistic programs sampling the space of probabilistic programs.This is a particularly important development for program learning.

A recent work on learning probabilistic programs within the "sampling the samplers" paradigm by Perov and Wood allows, in particular, "compilation" of probabilistic programs so that the resulting samplers just sample the posterior directly without sampling the whole joint distribution (another possible name for this procedure which comes to mind is "partial evaluation", although neither term is quite adequate for this novel procedure). The work is done using the new Anglican engine which implements a probabilistic programming language similar to Venture, but is written in Clojure (which should enable better parallelization and better performance scaling with more hardware) and uses a higher-order PMCMC ("Particle Markov Chain Monte Carlo") sampling scheme, where efficient high-dimensional proposal distributions for MCMC are generated by particle filters. The work follows earlier successes of Maddison and Tarlow in capturing frequent context-dependent syntactic patterns of code from open source repositories within generative models (see [33] and references therein). 
Another important work done with the use of multilevel "generative models emitting generative models" architecture is the research by Lake in compositional concept learning [24]. The typical tasks performed are learning the letters of synthetic alphabet and spoken Japanese-like words. The author claims that this is the first time when a machine learning system combines learning from one or a few examples (rather than from big data corpora) with learning rich conceptual representations.

\section{Partial Inconsistency, Non-monotonic Inference, and Vector Se- mantics}

The traditional mathematical view is that there is only one kind of contradiction and that all contradictions imply each other and everything else. However, there is also rich tradition of studying various kinds of graded or partial contradictions.

There are a number of common motives appearing multiple times in various studies of graded inconsistency. These common motives link a variety of independently done studies together and serve as focal elements of what we call the partial inconsistency landscape [5]. We list many of these common motives and some of their interplay.

An especially important motive is that in the presence of partial inconsistency many otherwise impoverished algebraic structures become groups and vector spaces. In particular, domains for denotational semantics tend to acquire group and vector space structure when partial inconsistency is present.

Known applications include handling of inconsistent information and non-monotonic and anti-monotonic inference. Perhaps even more importantly for the advanced AI, vector semantics is likely to offer new powerful schemes for program learning, as we are arguing in this paper.

We provide a necessarily incomplete overview of this field here and present some of our results. For more details, see [6] and other materials in [3].

\subsection{Focal Elements of the Partial Inconsistency Landscape}

- Various forms of negative measure (negative length and distance, negative probability and signed measures, negative membership and signed multisets)

- Bilattices

- Bitopology

- Domains with group and vector space structures

- Bicontinuous domains

- The domain of arrows, $D^{O p} \times D$ or $C^{O p} \times D$

- Non-monotonic and anti-monotonic inference

- Modal and paraconsistent logic and possible world models

- Hahn-Jordan decomposition or "bilattice pattern": $x=(x \wedge 0)+(x \vee 0)$ or $x=(x \wedge \perp) \sqcup(x \vee \perp)$

\subsection{Partially Inconsistent Interval Numbers}

Interval numbers are segments $[a, b]$ on the real line where $a \leq b$. One can extend interval numbers by adding pseudosegments $[a, b]$ with the contradictory property that $b<a$. This structure was independently discovered many times and is known under various names including Kaucher interval arithmetic, 
directed interval arithmetic, generalized interval arithmetic, and modal interval arithmetic (a comprehensive repository of literature on the subject is maintained by Evgenija Popova [34]). The first mention known to us is by Warmus in 1956 [40]. Our group tends to call this structure partially inconsistent interval numbers.

There are two partial orders on partially inconsistent interval numbers. The informational order, $\sqsubseteq$, is defined by reverse inclusion on interval numbers: $[a, d] \sqsubseteq[b, c]$ iff $a \leq b$ and $c \leq d$. The same formula is used for partially inconsistent interval numbers. The material order is component-wise: $[a, b] \leq[c, d]$ iff $a \leq c$ and $b \leq d$.

Addition on interval numbers (and partially inconsistent interval numbers) is defined componentwise: $\left[a_{1}, b_{1}\right]+\left[a_{2}, b_{2}\right]=\left[a_{1}+a_{2}, b_{1}+b_{2}\right]$.

The operation of weak minus is defined as $-[a, b]=[-b,-a]$. Addition and weak minus are monotonic with respect to $\sqsubseteq$.

Consider $-[a, b]+[a, b]=[-b,-a]+[a, b]=[a-b, b-a]$. If $a<b$, then the strict inequality, $[a-b, b-a] \sqsubset[0,0]$, holds. So if $a<b,-[a, b]+[a, b]$ approximates $[0,0]$, but is not equal to it, hence interval numbers with weak minus don't form a group.

If one allows pseudosegments, one can define the component-wise true minus: $-[a, b]=[-a,-b]$. Partially inconsistent interval numbers with the component-wise addition and the true minus form a group (and a $2 \mathrm{D}$ vector space over the reals). The true minus maps precisely defined numbers, $[a, a]$, to precisely defined numbers, $[-a,-a]$. Other than that, the true minus maps segments to pseudosegments and maps pseudosegments to segments. The true minus is anti-monotonic with respect to $\sqsubseteq$.

\subsection{Bilattices}

A bilattice is a set equipped with two lattice structures defining two partial orders, the material order, $\leq$, and the informational order, $\sqsubseteq$, and Ginsberg involution ${ }^{1}$ monotonic with respect to $\sqsubseteq$, anti-monotonic with respect to $\leq$, and preserving appropriate lattice structures. Additional axioms are often imposed.

Bilattices were introduced by Matthew Ginsberg [17] to provide a unified framework for a variety of inferences schemes used in AI, such as non-monotonic inference, inference with uncertainty, etc. They are now ubiquitous in the studies of partial and graded inconsistency.

The simplest example of a bilattice is the four-valued logic: $f<\perp<t, f<\top<t, \perp \sqsubset f \sqsubset \top, \perp \sqsubset$ $t \sqsubset \top$.

Partially inconsistent interval numbers form a bilattice. Sometimes one wants both orders to form complete lattices. This can be achieved by allowing $a$ and $b$ to also take $-\infty$ and $+\infty$ as values, or by confining $a$ and $b$ within a segment $[A, B]$, in both cases sacrificing the property of partially inconsistent interval numbers being a group.

If we consider all partially inconsistent interval numbers without infinities or allow $a$ and $b$ to take $-\infty$ and $+\infty$ values, or if we confine $a$ and $b$ within segment $[-A, A]$, then Ginsberg involution is the weak minus. If we confine $a$ and $b$ within a segment $[A, B]$, then Ginsberg involution maps $[a, b]$ to $[A+B-b, A+B-a]$. One important case here is $[A, B]=[0,1]$.

\subsection{Bitopology and Non-monotonic Inference}

Asymmetric topology such as Scott topology generated by a partial order $\sqsubseteq$ is often used in computer science to encode monotonic inference and limits of monotonic inference. For example, the upper topology on the real line consists of the open rays $(a,+\infty)$ (take $a=-\infty$ and $a=+\infty$ to represent the whole space and the empty set). This topology encodes the processes generating monotonically non-decreasing sequences of reals, $x_{1} \leq x_{2} \leq \ldots$, and their limits.

${ }^{1}$ an involution is a function $f$ such that $f(f(x))=x$ for all $x$ in the domain of $f$ 
Scott continuous functions are functions respecting this structure. More specifically, Scott continuous functions between two spaces with Scott topologies are monotonic functions preserving appropriately defined limits. A classic exposition of these ideas is [37].

For an exposition of inference in Scott domains see, for example, Chapter 5 of [4]. Because Hasse diagrams depict partially ordered sets in such a fashion that the larger elements are above the smaller elements, we say that the standard monotonic inference in Scott domains is directed upwards (the elements become larger in the process of inference).

If there are two Scott topologies on the same set with associated partial orders pointing into opposite directions, one can infer both upwards and downwards, thus enabling non-monotonic inference.

In our example, one can also consider the lower topology on the real line consisting of the open rays $(-\infty, b)$, and this is the second Scott topology, encoding the processes generating monotonically nonincreasing sequences of reals, $y_{1} \geq y_{2} \geq \ldots$, and their limits. Switching between these two topologies one can encode non-monotonic sequences.

A space with two topologies is called a bitopological space, and a space with Scott topologies generated by $\sqsubseteq$ and $\sqsupseteq$ with certain additional properties is called a bicontinuous domain [21].

\subsection{Order Reversal and the Domain of Arrows}

Consider a bicontinuous domain, $(X, \sqsubseteq, \sqsupseteq)$. The partial order $\sqsupseteq$ which is the order opposite to $\sqsubseteq$ then defines the dual space, $X^{O p}=X^{*}=(X, \sqsupseteq, \sqsubseteq)$, which is also a bicontinuous domain.

For a bicontinuous domain $(X, \sqsubseteq, \sqsupseteq)$ we say that $\sqsubseteq$ is pointing upwards ("the main partial order of the space") and $\sqsupseteq$ is pointing downwards ("the auxiliary or dual order of the space").

If we think informally about an arrow from space $X$ to space $Y$, then our intuition tells us that the arrow is greater if it "points more upwards", that is, if its right end is higher, and its left end is lower.

Formalizing this intuition we define the space of arrows from $X$ to $Y$ as $X^{*} \times Y$.

If we consider real numbers $\mathbb{R}$ with the standard order, $\sqsubseteq=\leq$, then partially inconsistent interval numbers are a space of arrows pointing from the right ends of the segments to the left ends of the segments, $\mathbb{R} \times \mathbb{R}^{*}$.

If $\mathbb{R}$ is modified to become a domain, $R$ (by adding $-\infty$ and $+\infty$ as values or by taking a finite segment), we call $R \times R^{*}$ a domain of arrows.

There are two ways to describe Scott topology in terms of generalized distances. One is via asymmetric quasi-metrics, with $d(x, y)=0$ if and only if $x \sqsubseteq y$. Another is via dropping the $d(x, x)=0$ requirement which leads to relaxed and partial metrics. Quasi-metrics of this kind are monotonic with respect to one of the variables and anti-monotonic with respect of another variable. So the only way to have these generalized distances to be Scott continuous as functions from $X \times X$ to the domain representing distances is via the route of relaxed and partial metrics [11].

In the bicontinuous situation, quasi-metrics can be understood as (Scott continuous) order-preserving maps from the domain of arrows, $X^{*} \times X$, to the domain representing distances.

Order-reversing involutions $(x \sqsubseteq y \Leftrightarrow f(y) \sqsubseteq f(x)$ and $f(f(x))=x$ ) play a prominent role in this context. From the viewpoint of domain theory, order-reversing involutions should be thought of as order-preserving maps $X \rightarrow X^{*}$ (or $X^{*} \rightarrow X$ ). Hence order-reversing involutions are order-preserving maps $X^{*} \times X \rightarrow X \times X^{*}$ (and vice versa) on the domain of arrows.

\subsection{Bitopology and Partial Inconsistency}

There are at least three ways bitopologies occur in studies of partial inconsistency. The connections between partial inconsistency and bitopological Stone duality via the notion of $d$-frame (Jung-Moshier frame) are explored in [20] (see also [25]). A fuzzy bitopology valued in lattice $L$ is a fuzzy topology 
valued in the bilattice $L^{2}$ (in particular, an ordinary bitopology is a topology valued in the four-valued logic) [36]. Finally, in the context of bitopological groups and the anti-monotonic group inverse the following situation is typical: two topologies, $T$ and $T^{-1}$, are group dual of each other (i.e. the group inverse induces a bijection between the respective systems of open sets), the multiplication is continuous with respect to both topologies, and the group inverse is a bicontinuous map from $\left(X, T, T^{-1}\right)$ to its bitopological dual, $\left(X, T^{-1}, T\right)[1]$.

All these motives are prominent for the case of partially inconsistent interval numbers [6].

D-frames. Partially inconsistent interval numbers over reals extended with $\pm \infty$ are isomorphic to the $d$-frame of the (lower, upper) bitopology on the reals.

Consider the (lower, upper) bitopology on the real line, that is the bitopology where the first topology is the lower topology, and the second topology is the upper topology (see Section 4.4). Define the bilattice isomorphism between the d-frame elements, i.e. pairs $\langle L, U\rangle$ of the respective open sets, and partially inconsistent interval numbers. A pair $\langle L, U\rangle$ is a pair of open rays, $\langle(-\infty, a),(b,+\infty)\rangle(a$ and $b$ are allowed to take $-\infty$ and $+\infty$ as values). This pair corresponds to a partially inconsistent interval number $[a, b]$. Consistent, i.e. non-overlapping, pairs of open rays $(a \leq b)$ correspond to segments. Total, i.e. covering the whole space, pairs of open rays $(b<a)$ correspond to pseudosegments.

Group dual topologies. The minus operation on real numbers is bicontinuous from the (lower, upper) bitoplogy to the (upper, lower) bitopology and vice versa. The corresponding map between the $d$ frames is very similar to the weak minus (Ginsberg involution), except that the order of bitopological components also needs to be swapped to respect bitopological duality in this case (partially inconsistent interval numbers are a Cartesian product of lower and upper bounds; swapping can be thought of as changing the order of components in this Cartesian product).

In a similar fashion, the true minus operation on the partially inconsistent interval numbers is bicontinuous between a $\left(T, T^{-1}\right)$ bitopology on the partially inconsistent interval numbers and its dual $\left(T^{-1}, T\right)$ bitopology. (Here $T$ and $T^{-1}$ must be group dual topologies of each other, e.g. the Scott topology corresponding to $\sqsubseteq$ and the Scott topology corresponding to $\sqsupseteq$.)

Rodabaugh correspondence. Any real-valued fuzzy bitopology can be represented as fuzzy topology valued in partially inconsistent interval numbers.

The open sets of the upper topology on the reals is a particular representation of real numbers extended with $\pm \infty$, the same is true about the open sets of the lower topology on the reals. Consider a particular form of real-valued fuzzy bitopology, namely the multivalued bitopology where the first multivalued topology is valued in the lower topology, and the second multivalued topology is valued in the upper topology, that is a (lower, upper)-valued bitopology.

Consider the following mild generalization of the correspondence described in [36]. $(L, M)$-valued bitopology can be understood as $L \times M$-valued topology via $L^{X} \times M^{X} \cong(L \times M)^{X}$ isomorphism.

Hence the (lower, upper)-valued bitopology can be understood as the topology valued in the (lower, upper) d-frame, i.e. the topology valued in partially inconsistent interval numbers over reals extended with $\pm \infty$. Further discussion of the intuition involved here is in the slides 37-38 of [6].

\subsection{Paraconsistent Version of Fuzzy Mathematics}

It seems that mathematics of partial inconsistency should be bilattice-valued. The Rodabaugh correspondence is one of the indications of that, as $L \times M$ is naturally a bilattice, with the informational order, $\sqsubseteq_{L \times M}$, being obtained from the product $\left(L, \sqsubseteq_{L}\right) \times\left(M, \sqsubseteq_{M}\right)$ and the material order, $\leq_{L \times M}$, being obtained from the product of $L$ by the dual of $M,\left(L, \sqsubseteq_{L}\right) \times\left(M, \sqsupseteq_{M}\right)$. 
While the fuzzy mathematics in general is lattice-valued, the situations where the lattice is $[0,1]$ or otherwise based on real numbers remain important. Similarly, while mathematics of partial inconsistency is in general likely to be valued in bilattices, the particular situations where the bilattice is based on partially inconsistent real numbers (whether confined within $[0,1],[-1,1]$, or $[-\infty,+\infty]$ ) are likely to play important roles.

The paraconsistent equivalent of real-valued fuzzy mathematics is mathematics valued in partially inconsistent interval numbers.

\subsection{Partial and Relaxed Metrics}

The standard partial metric on the interval numbers is $p\left(\left[a_{1}, b_{1}\right],\left[a_{2}, b_{2}\right]\right)=\max \left(b_{1}, b_{2}\right)-\min \left(a_{1}, a_{2}\right)$ [8]. Hence the self-distance for $[a, b]$ is $b-a$. If we extend this formula to pseudosegments, the self-distance of pseudosegments turns out to be negative.

Partial metrics can be understood as upper bounds for "ideal distances". One often has to trade the tightness of those bounds for nicer sets of axioms. E.g. the natural upper bound for the distance between $[0,2]$ and $[1,1]$ is 1 , and there is a weak partial metric which yields that. However, if one wants to enjoy the axiom of small self-distances, $p(x, x) \leq p(x, y)$, one has to accept $p([0,2],[1,1])=2$, since $p([0,2],[0,2])=2$.

A similar trade can be made for lower bounds. The standard interval-valued relaxed metric produces the gap between non-overlapping segments as their lower bound, but takes 0 as the lower bound for the distance between overlapping segments (hence 0 is also the lower bound for self-distance). If one settles for a less tight lower bound and allows the lower bound to be negative in those cases, one can obtain a distance with much nicer properties: $l\left(\left[a_{1}, b_{1}\right],\left[a_{2}, b_{2}\right]\right)=\max \left(a_{1}, a_{2}\right)-\min \left(b_{1}, b_{2}\right)$.

We think about the pair $\langle l, p\rangle$ as a relaxed metric valued in partially inconsistent interval numbers. The self-distance of $[a, b]$ is $[a-b, b-a]$ and the self-distance of a pseudosegment is a pseudosegment.

The map $[a, b] \mapsto[b, a]$ expressing the symmetry between segments and pseudosegments also transforms $\langle l, p\rangle$ into $\langle p, l\rangle$.

\subsection{Signed Measures and Signed Multisets}

One way to think about $p([a, b],[a, b])=b-a$ is to say that a pseudosegment has a negative length.

We can also revisit the correspondence between the elements of the (lower, upper) bitopology dframe, $\{\langle(-\infty, a),(b,+\infty)\rangle\}$, and the partially inconsistent interval numbers. Consider the characteristic function mapping the real line to 1 and subtract from it the characteristic functions of $(-\infty, a)$ and $(b,+\infty)$. If $[a, b]$ is a segment, the result is the characteristic function of that segment (valued 1 for the points belonging to the segment and 0 for the points outside the segment). If $[a, b]$ is a pseudosegment and if we allow for the overlap between $(-\infty, a)$ and $(b,+\infty)$ to be subtracted twice, the result is the generalized characteristic function, which equals to -1 in the open interval $(b, a)$ and equals to 0 outside $(b, a)$. So we obtain a signed multiset here allowing negative degree of membership.

This construction is topologically asymmetric in the following sense. Algebraically we can say that totally defined numbers $[a, a]$ belong to both segments and pseudosegments, or to neither. But topologically (and via characteristic functions), this symmetry must be broken. We break it in favor of the "natural" viewpoint: totally defined numbers are segments, and not pseudosegments. But one could also break it in favor of the dual viewpoint, by considering dual d-frames of closed sets (and stipulating that characteristic functions of segments take value 1 only on their interiors). 


\subsection{Negative Probability and Vector Semantics}

One can think about probabilistic programs as transformers from the probability distributions on the space of inputs to the probability distributions on the space of outputs. Dexter Kozen showed that it is fruitful to replace the space of probability distributions by the space of signed measures [23]. One defines $v<\mu$ iff $\mu-v$ is a positive measure. The space of signed measures is a vector lattice (a Riesz space) and a Banach space, so people call this structure a Banach lattice. Denotations of programs are continuous linear operators with finite norms. The probabilistic powerdomain is embedded into the positive cone of this Banach lattice. The structure of Hilbert space on signed measures can be obtained via reproducing kernel methods (see Chapter 4 of [2]).

\subsection{Hahn-Jordan Decomposition and the Bilattice Pattern: $x=(x \wedge 0)+(x \vee 0)$ or $x=(x \wedge \perp) \sqcup(x \vee \perp)$}

The Hahn-Jordan decomposition, $\mu^{+}=\mu \vee 0, \mu^{-}=\mu \wedge 0, \mu=\mu^{+}+\mu^{-}$, holds, due to the fact that $x=(x \wedge 0)+(x \vee 0)$ is a theorem for all lattice-ordered groups.

Defining $v \sqsubseteq \mu$ iff $v^{+} \leq \mu^{+}$and $v^{-} \leq \mu^{-}$, one also obtains $\mu=\mu^{+} \sqcup \mu^{-}$, making this an instance of the "bilattice pattern", $x=(x \wedge \perp) \sqcup(x \vee \perp)$. The "bilattice pattern" appears independently in a variety of studies on partial inconsistency [5].

It looks like the right degree of generality here might be lattice-ordered monoids with an extra axiom, $x=(x \wedge 0)+(x \vee 0)$.

\subsection{Possible Worlds Indexed by Measures}

William Wadge explored various ways to index possible worlds in the context of intensional logic and data flow programming [39]

In our case, possible worlds would be indexed by measures, which is quite attractive and feels natural: a world is distinguished by how often one observes various phenomena, and we do sampling observations to figure out what kind of world we currently inhabit.

In a classical situation one would normally consider probability measures for this role, but in a quantum situation signed quasi-probability distributions or complex-valued amplitudes would naturally play this role.

\subsection{Distances Between Programs}

On one hand, Anthony Seda and Máire Lane note that there is a natural norm for Kozen semantic spaces, which allows us to define a conventional metric on program denotations and hence a conventional distance between programs [38].

On the other hand, in the context where everything is a function of a measure (possible worlds are indexed by measures), the standard constructions of generalized distances (partial metrics, relaxed metrics, and quasi-metrics) over Scott domains which tend to be parametrized by measures (see, for example, Section 7 of [7]) look quite natural.

We tend to view the dependency of those constructions on a measure as an obstacle which needs to be overcome, but perhaps it is actually a desirable feature.

\subsection{Computational Models with Involutions}

We are currently looking at various computational models involving involutions. 
Given a domain of arrows $X \times X^{*}$, a sequence $\left(x_{1}, y_{1}\right),\left(x_{2}, y_{2}\right), \ldots$ is called a monotonic sequence with involutive steps, if for any $n \in \mathbb{N}$ either $x_{n} \sqsubseteq x_{n+1}$ and $y_{n} \sqsupseteq y_{n+1}$ (in which case the step $n$ is called monotonic) or $x_{n}=y_{n+1}$ and $y_{n}=x_{n+1}$ (in which case the step $n$ is called an involution).

One can define a notion of convergence robust with respect to the insertion of pairs of involutive steps and prove that if $(x, y)$ is a limit under this notion, then $x=y$.

Architectures based on involutive steps are rather prominent in the context of reversible and quantum computations. For example, the well-known Grover's quantum algorithm can be described as a sequence of reflections of subsets of a plane [35].

Architectures where the state of an abstract machine is an image on the plane, and an involutive computational step selects a line on this plane and a subset symmetric with respect to this line, and performs a reflection of the image within this subset, seem to be quite attractive in the context of classical computations as well.

\section{Conclusion}

It is possible to talk about linear combinations of probabilistic programs when their semantics is expressed as linear operators [23].

For $0<\alpha<1$ and random being a generator of uniformly distributed reals between 0 and 1 , the linear operator corresponding to the program if random $<\alpha$ then $\mathbf{P}$ else $\mathbf{Q}$ is a linear combination of the linear operators corresponding to programs $\mathbf{P}$ and $\mathbf{Q}$ with coefficients $\alpha$ and $1-\alpha$.

However, when one aims for better schemes of program learning, the situations where one can consider linear combinations of single execution runs rather than linear combinations of the overall program meanings should be especially attractive. In this paper we consider two such architectures, probabilistic sampling and generalized animation, and the recent progress in this field looks very promising.

We give an overview of mathematical material tightly connected to linear models of computations via the partial inconsistency landscape. Vector semantics is an integral part of the partial inconsistency landscape, and we expect that other key elements of that landscape will be finding more uses as linear models of computations and their applications are further explored.

We would like to conclude by describing a possible hybrid approach to program learning. Instead of implementing everything in terms of architectures admitting linear combinations of single execution runs one can use a hybrid approach, mixing these architectures and traditional software. In this context we might be inspired by hybrid hardware connecting live neural tissue and electronic circuits.

One might decide to use large existing software components and try to automate the process of connecting them together using flexible probabilistic connectors. Here one should note the progress in automated generation of test suites for software systems.

Another hybrid approach involves the use of small inflexible components inside the flexible "tissue" of linear models. Our experiments in dataflow programming with streams supporting the notion of a linear combination of streams described in Sections 1.4, 1.5 are examples of this hybrid approach $[9,10]$. In particular, the template operations play the role of small inflexible components in [10], where dataflow graphs are represented by matrices of real numbers describing the flexible connectivity patterns from the outputs to the inputs of a potentially countable number of template operations.

Acknowledgments. We would like to thank Ralph Kopperman, Lena Nekludova, Leon Peshkin, Josh Tenenbaum, and Levy Ulanovsky for helpful discussions. 


\section{References}

[1] S. Andima, R. Kopperman, and P. Nickolas. An asymmetric Ellis theorem. Topology and Its Applications, 155:146-160, 2007.

[2] A. Berlinet and C. Thomas-Agnan. Reproducing Kernel Hilbert Spaces in Probability and Statistics. Kluwer Academic Publishers, Boston, 2004.

[3] M. Bukatin. Partial inconsistency and vector semantics of programming languages (a repository of research materials). http://www.cs.brandeis.edu/ bukatin/partial_inconsistency.html.

[4] M. Bukatin. Mathematics of Domains. PhD thesis, Brandeis University, 2002. http://www . cs . brandeis . edu/ bukatin/Mathematics-of-Domains.pdf.

[5] M. Bukatin, R. Kopperman, and S. Matthews. Partial inconsistency landscape: an overview. 28th Summer Conference on Topology and Its Applications, Nipissing University, July, 2013. http://www . cs . brandeis . edu/ bukatin/PartialInconsistencyJul24.pdf.

[6] M. Bukatin, R. Kopperman, and S. Matthews. Progress report on partial inconsistency, bitopology, and vector semantics. Conference on Computational Topology and Its Applications, Kent State University, Ohio, November 2014. http://www.cs . brandeis . edu/ bukatin/PartialInconsistencyProgressNov2014 . pdf.

[7] M. Bukatin, R. Kopperman, and S. Matthews. Some corollaries of the correspondence between partial metrics and multivalued equalities. Fuzzy Sets and Systems, 256:57-72, 2014.

[8] M. Bukatin, R. Kopperman, S. Matthews, and H. Pajoohesh. Partial metric spaces. American Mathematical Monthly, 116:708-718, 2009.

[9] M. Bukatin and S. Matthews. Almost continuous transformations of software and higher-order dataflow programming, Preprint (2015). http://www .cs . brandeis .edu/ bukatin/HigherOrderDataFlow . pdf.

[10] M. Bukatin and S. Matthews. Dataflow graphs as matrices and programming with higher-order matrix elements, Preprint (2015). http://www . cs.brandeis.edu/ bukatin/DataFlowGraphsAsMatrices .pdf.

[11] M. Bukatin and J. Scott. Towards computing distances between programs via Scott domains. In S. Adian and A. Nerode, editors, Logical Foundations of Computer Science, LNCS, vol. 1234, pages 33-43. Springer, 1997.

[12] S. Carrol. Evo-devo and an expanding evolutionary synthesis: A genetic theory of morphological evolution. Cell, 134:25-36, 2008.

[13] S. Draves. The Electric Sheep screen-saver: A case study in aesthetic evolution. In F. Rothlauf et al., editors, Applications of Evolutionary Computing, LNCS, vol. 3449, pages 458-467. Springer, 2005. http: //draves.org/evomusart05/.

[14] R. Feynman. Negative probability. In F. Peat and B. Hiley, editors, Quantum Implications : Essays in Honour of David Bohm, pages 235-248. Routledge and Kegan Paul, 1987. http://cds . cern . ch/record/154856/ files/pre-27827.pdf.

[15] Fluid. Project "Fluid" github repository, 2015. https://github.com/anhinga/fluid.

[16] J. Gerhart and M. Kirschner. The theory of facilitated variation. Proc. Natl. Acad. Sci., 104(Suppl 1):85828589, 2007.

[17] M. Ginsberg. Multivalued logics: a uniform approach to inference in artifcial intelligence. Computational Intelligence, 4(3):256-316, 1992.

[18] H. Groenewold. On the principles of elementary quantum mechanics. Physica, 12:405-460, 1946.

[19] R. Grosse, R. Salakhutdinov, W. Freeman, and J. Tenenbaum. Exploiting compositionality to explore a large space of model structures, 2012. http://arxiv.org/abs/1210.4856.

[20] A. Jung and M.A. Moshier. On the bitopological nature of Stone duality. Technical Report CSR-06-13, School of Computer Science, University of Birmingham, 2006. http://www.cs.bham.ac.uk/ axj/pub/ papers/Jung-Moshier-2006-On-the-bitopological-nature-of-Stone-duality.pdf.

[21] K. Keimel. Bicontinuous domains and some old problems in domain theory. Electronic Notes in Theoretical Computer Science, 257:35-54, 2009.

[22] M. Kirschner and J. Gerhart. The Plausibility of Life: Resolving Darwin's Dilemma. Yale University Press, 
2005.

[23] D. Kozen. Semantics of probabilistic programs. Journal of Computer and System Sciences, 22(3):328-350, 1981.

[24] B. Lake. Towards More Human-like Concept Learning in Machines: Compositionality, Causality, and Learning-to-learn. PhD thesis, MIT, 2014. http://cims.nyu . edu/ brenden/LakePhDThesis .pdf.

[25] J. Lawson. Stably compact spaces. Mathematical Structures in Computer Science, 21(1):125-169, 2011.

[26] M. Looks. Competent Program Evolution. PhD thesis, Washington University in St. Louis, 2002. http: //metacog.org/doc.html.

[27] W. Maass. Noise as a resource for computation and learning in networks of spiking neurons. Proc. of the IEEE, 102(5):860-880, 2014

[28] V. Mansinghka, T. Kulkarni, Yu. Perov, and J. Tenenbaum. Approximate Bayesian image interpretation using generative probabilistic graphics programs, 2013. http://probcomp.csail.mit. edu/gpgp.

[29] D. Marr. Vision. W. H. Freeman and Company, New York, 1982.

[30] C. Miquel, J. Paz, and M. Saraceno. Quantum computers in phase space. Phys. Rev. A, 65(6):062309, 2002. http://arxiv.org/abs/quant-ph/0204149.

[31] J. Moyal. Quantum mechanics as a statistical theory. Proceedings of the Cambridge Philosophical Society, 45:99-124, 1949. http://physics.bu. edu/ youssef/quantum/moyal.pdf.

[32] M. Pelikan. Bayesian Optimization Algorithm: from Single Level to Hierarchy. PhD thesis, University of Illinois at Urbana-Champaign, 2002. http://www .medal-lab.org/files/2002023.pdf.

[33] Yu. Perov and F. Wood. Learning probabilistic programs, 2014. http://arxiv .org/abs/1407.2646.

[34] E. Popova. The arithmetic on proper \& improper intervals (a repository of literature on interval algebraic extensions). http://www.math.bas.bg/ epopova/directed.html.

[35] E. Rieffel and W. Polak. Quantum Computing: A Gentle Introduction. MIT Press, 2011.

[36] S. Rodabaugh. Functorial comparisons of bitopology with topology and the case for redundancy of bitopology in lattice-valued mathematics. Applied General Topology, 9(1):77-108, 2008.

[37] D. Scott. Outline of a mathematical theory of computation. Technical Report PRG02, Oxford University Computing Laboratory, 1970. https://www.cs.ox.ac.uk/files/3222/PRG02.pdf.

[38] A. Seda and M. Lane. On continuous models of computation: towards computing the distance between (logic) programs. In J. Morris, B. Aziz, and F. Oehl, editors, 6th International Workshop on Formal Methods (IWFM'03 Proceedings). Dublin City University, 2003. http://www.bcs.org/upload/pdf/ewic iwfm03_paper1.pdf.

[39] W. Wadge. Intensional logic in context. In M. Gergatsoulis and P. Rondogiannis, editors, Intensional Programming II: based on the papers at Islip 99, pages 1-13. World Scientific, 2000. http://www . cse . unsw . edu.au/ plaice/archive/ISLIP/ip2/ip2_001.pdf.

[40] M. Warmus. Calculus of approximations. Bull. Acad. Pol. Sci., Cl. III, 4(5):253-259, 1956. http://www . cs.utep.edu/interval-comp/warmus.pdf. 